\title{
Selection of Route of Temporary Irrigation Water Supply Line with GIS in Emergency Situation
}

\author{
Akira Kobayashi ${ }^{1}$, Kiyohito Yamamoto ${ }^{2}$ and Takuma Hayashi ${ }^{3}$
}

\begin{abstract}
In natural disasters, the agricultural activity sometimes suffers enormous damage such as failure of an irrigation tank and a water channel. To produce an agricultural commodity in such emergency circumstances, water has to be supplied by temporary works. In many cases, the water supply line is temporarily built from undamaged tanks or head works. In this paper, the selection method of the temporary irrigation water supply line with GIS was presented. The digital elevation model having $2.5 \mathrm{~m}$ resolution was used in GIS. The functions of GIS software were used as much as possible because the method has to be easily and quickly operated. Since the facility has to be constructed economically and rapidly, it was assumed that the water supply line would be constructed on the ground surface without excavation works and the gravity irrigation of a closed type pipeline would be adopted. For the siphon design, the location giving the high negative pressure was avoided. To examine the selected routes, the indexes indicating the ratio of positive pressure, the length of the line and the undulation of the route were introduced. It was found that the method using the information of elevation gave the economic route and the method using the gradient and slope direction information gave the safe and easy constructible route.
\end{abstract}

\section{Keywords: Temporary work; Water supply line; GIS; Route selection; Disasters; Hydraulic analyses}

\section{Introduction}

In 2004, ten typhoons hit Japan. One of them hit directly the Awaji Island. Over 180 irrigation tanks in the island were failed by overflow due to heavy downpour (Japanese Society of Irrigation, Drainage and Reclamation Engineering, Kyoto branch, 2005). Many floods due to heavy downpour have occurred at various places in Japan every year. In 2004 and 2007, huge earthquakes occurred in Niigata prefecture. Tohoku and Kyusyu districts suffered from earthquakes in 2005. Ishikawa prefecture also suffered from a large earthquake in 2007. Japan enters a period of brisk seismic activity. Probability of such enormous disasters seems large during recent years. Such disasters induced failures of many agricultural facilities, and agricultural activities sustained huge damages from such disasters. For such an emergency situation, the usual maintenance of the facility is very important. Inoue et al. (2007) has developed the history management system using GIS for a large scale agricultural pipeline. By using this system, the systematic maintenance can be carried out. However, regardless of the usual maintenance, the probability of the failure of the facility due to a huge disaster is high. For the rehabilitation of national irrigation facilities damaged by the earthquake at Niigata prefecture in 2004, a total of 5.88 hundred million yen was expensed (Naka et al., 2006).

To produce an agricultural commodity in such emergency circumstances, firstly water has to be secured. In the disaster in Niigata in 2004, since water was supplied later

\footnotetext{
${ }^{1}$ Associate Professor, Graduate School of Agriculture, Kyoto University, Kitashirakawa-oiwake-cho, Sakyo-ku, Kyoto 606- 8502, Japan (Corresponding Author) E-mail: kobadesu@kais.kyoto-u.ac.jp

${ }^{2}$ Assistant, Graduate School of Agriculture, Kyoto University, Kitashirakawa-oiwake-cho, Sakyo-ku, Kyoto 606- 8502, Japan

${ }^{3}$ Student, Graduate School of Agriculture, Kyoto University, Kitashirakawa-oiwake-cho, Sakyo-ku, Kyoto 606-8502, Japan
}

by one month in 2005 than usual despite of the rapid rehabilitation, the production was reduced to about $80 \%$ of the average production due to the damage from a pest. The temporary facilities have an important role to secure the productivity. In many cases, a pipeline or a water hose has been used as temporary works to run water from undamaged tanks or head works. Photo 1 shows the photograph of a temporary water line used in Niigata (Niigata prefecture homepage). However, to run water safely though a tube, some conditions have to be satisfied. Moreover, a short pipeline is preferable from the economic point of view. Although such a facility is temporary, recovery of main facilities needs time of a few years in some cases. Therefore, safety of temporary facilities has to be examined well.

In this paper, the method to select the route of a temporary irrigation water supply line with GIS is presented. As a selection of the route of an irrigation pipeline, Tanaka et al. (2002) developed the object oriented system using an interactive interface, while that is the system for a large scale pipeline. There are some restrictions for the temporary irrigation water line. The facility has to be economical and be constructed rapidly. Therefore, the complicated system used for a general irrigation pipeline is not suited. In this study, the gravity irrigation of the closed type pipeline is assumed to be adopted. Moreover, the management of water is also simple and the operation by a user is not considered. Thus, since the adjustment system is not needed, the analysis of water hammer and non-steady analysis are also neglected in this study. The steady state of a water pressure is considered to avoid the obstruction of the water flow in the tube.

The temporary water line is assumed to be built on the ground surface. Excavation works are not carried out from the economic and time constraints. Therefore, the route is 
selected by using the elevation data of the region. In this study, the digital elevation model (DEM) having fine resolution is applied to GIS. As software of GIS, ArcGIS (Scott et al., 2003) is used. In the software, the cost weighted distance function of Spatial Analyst is used to select the route. The cost weighted distance is the distance considering the cost such as the transport time or expenses. The route having the lowest cost is determined by this function as explained later. As a cost, the elevation information and the slope information are used in this study. Moreover, the combination of both costs is also examined. Totally three methods are examined to determine the route of the gravity irrigation of the closed type pipeline. To evaluate the route quickly, it is effective that the function incorporated in the software has to be used as much as possible. Moreover, to examine the selected routes, the length of the route, the ratio of the route under the hydraulic gradient line and the workability of the construction are compared for three different methods.

\section{High resolution satellite image DEM}

For an actual disaster, an aerial photograph has to be prepared after the disaster because the topography may be changed. DEM also has to be obtained from the aerial photograph. In this study, DEM obtained by a satellite image is used as information on elevation. By a stereoscope technology with two satellite images, the distribution of elevation with resolution of $2.5 \mathrm{~m}$ is obtained for the area of $5 \mathrm{x}$ $5 \mathrm{~km}$. The information is incorporated into GIS. Figure 1 shows the image examined in this study. In the figure, starting and arrival points of a water supply line are also indicated. Although this image is the one of Awaji Island, the study in this paper is not related to the actual disaster in 2004. The locations of the starting and arrival points of irrigation water supply lines do not coincide with the locations of actual agricultural facilities. Hypothetical locations are selected for the study. Figure 2 indicates the bird view of geography of the area. This area includes the highest mountain in the island. Therefore, the location at the high elevation has many steep slopes.

\section{Analysis method}

\subsection{Cost weighted distance}

The function of the cost weighted distance of GIS software is used to analyze the route of an irrigation water supply line. Firstly, the method to calculate the cost weighted distance is explained in this section. The cost weighted distance is calculated by multiplying the distance by the averaged cost of the adjoining cells. For example, the cost weighted distance of two cells shown in Figure 3 is calculated. The cost is illustrated in each cell. The length of a side is 1 and the shape is a square. For Figure 3 (a), the average cost is 3 and then the cost weighted distance is obtained as $1 \times 3=3$. For (b), the distance is $\sqrt{2}$ and then the cost weighted distance becomes $\sqrt{2} \times 3=3 \sqrt{2}$.

The calculation starts from the starting point. The procedure starts from the cells surrounding the starting point

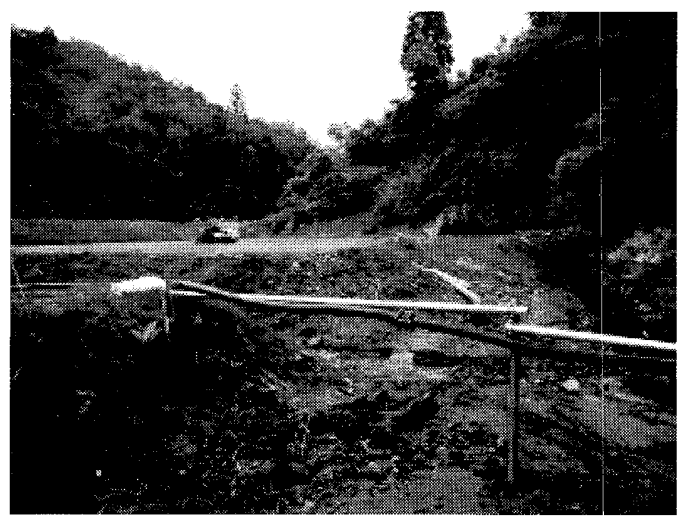

Photo 1: Temporary water supply line

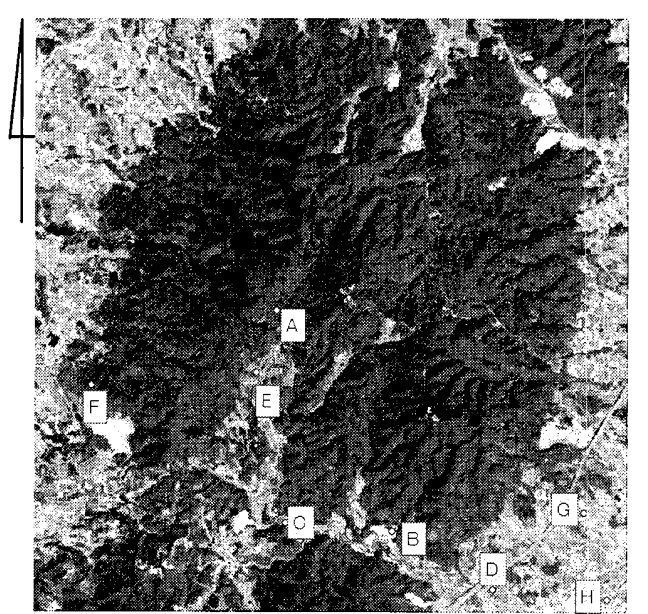

Figure 1: Examined area and location of starting and arrival points $(5 \times 5 \mathrm{~km})$

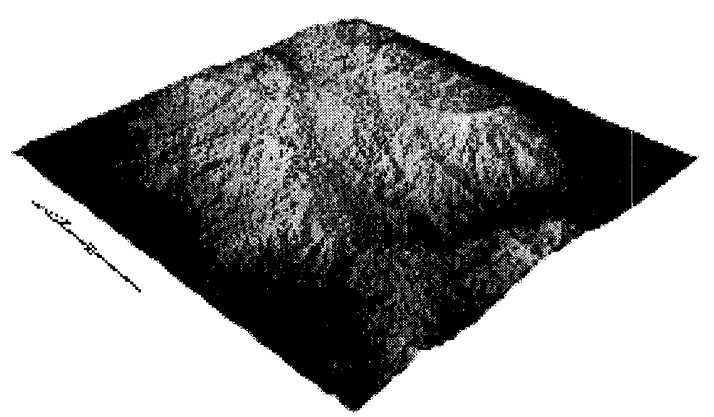

Figure 2: Bird view of examined area

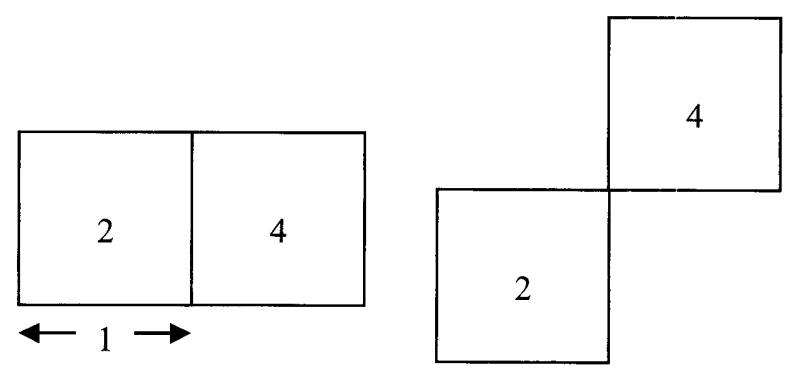

(a)

(b)

Figure 3: Calculation of cost weighted distance 
and then the cells departing from the starting point are treated in sequence. By using the example of Figure 4, the procedure is explained. The cost and the number of the cells are described in Figure 4 (a). The starting point is the cell at left down which is numbered as $(1,1)$. The arrival point is the cell of $(3,3)$. The cost weighted distance is obtained for the cells surrounding the cell of $(1,1)$. The value is registered in each cell as shown in Figure 4 (b). Then the cells secondly departing from the stating points are treated, which are $(1,3)$ and $(3,1)$. The cells adjoining to $(3,1)$ are $(2,1),(2,2)$. $(3,2)$ is not included because $(3,2)$ is farther than $(3,1)$ from the starting point. The cost weighted distance of $(3,1)$ is calculated with the cost of $(2,1)$ such that $1 \times(3+5) / 2=4$. That for $(2,2)$ is calculated such that $\sqrt{2} \times(5+7) / 2=8.5$. The accumulated value on the cost weighted distance of $(2,1)$ is obtained as $4+1.5=5.5$. That for $(2,2)$ is calculated as $4.95+8.5=13.45$. In the procedure, the smallest accumulated value is selected as the value of $(3,1)$. Thus, the value of 5.5 is registered into $(3,1)$ as shown in Figure 4 (b). Similarly, the accumulated value of $(1,3)$ is obtained as 5 that $1+1 \times(2+6) / 2=5$. The procedure is carried out until the arrival cell of $(3,3)$. The smallest accumulated cost weighted distance from the starting point to each cell is shown in Figure 4 (b). As a result, the smallest total cost from the starting cell to any cell can be known.

Then, the direction is registered in the cell. Figure 4 (c) shows the direction code. The direction of the cell giving the smallest accumulated cost weighted distance is indicated by the arrow in Figure 4 (b). The direction is registered with the direction code as shown in Figure 4 (d). By this information, the route having the smallest cost from any cell to the starting cell can be identified. In this study, this function is applied to select the route of an irrigation water supply line.

\subsection{Cost for route selection}

As a temporary facility, the maintenance and construction costs have to be small. While the facility is economical, the safety and the function of the facility have to be possibly secured. In this study, the route is selected with the assumption that the irrigation water supply line is constructed on the ground surface. Moreover, the system that water flows through the tube by the gravitational flow is examined. The location of the water intake is, therefore, selected at the position having a higher elevation than the location of water supply. Based on these fundamental assumptions, three values are used as a cost in the study; elevation and a pair of gradient and down slope direction. Moreover, the combination of both is used. In this paper, the term of "cost" is used as a value of a cell applied to calculate the cost weighted distance. To calculate the cost weighted distance of the function of GIS software, the cost has to be positive.

\subsubsection{Elevation cost}

By using the elevation as a cost, the route selecting the low location to the arrival point can be identified. As mentioned later, if a high negative pressure occurs in the route, a high

\begin{tabular}{|c|c|c|}
\hline 5 & 6 & 10 \\
$(3,1)$ & $(3,2)$ & $(3,3)$ \\
\hline 3 & 7 & 8 \\
$(2,1)$ & $(2,2)$ & $(2,3)$ \\
\hline 0 & 2 & 6 \\
$(1,1)$ & $(1,2)$ & $(1,3)$ \\
\hline
\end{tabular}

(a) Cost and cell number

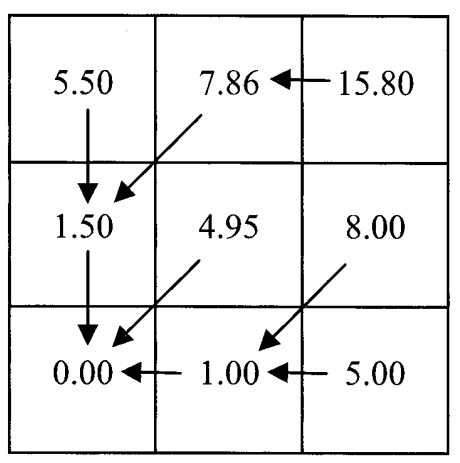

(b) Weighted distance

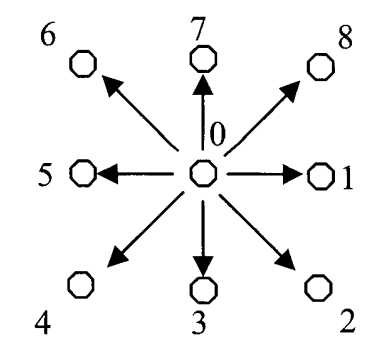

(c) Direction code

\begin{tabular}{|l|l|l|}
\hline 3 & 4 & 5 \\
\hline 3 & 4 & 4 \\
\hline 0 & 5 & 5 \\
\hline
\end{tabular}

(d) Registered direction code

Figure 4: Procedure of calculation of weighted distance and registration of direction code 
cost is imposed on the location having the negative pressure and then the selection is again tried. In the second trial, the location having the high negative pressure will be avoided. By this iteration procedure, it is expected that the route through which water runs smoothly by a gravitational flow is selected.

\subsubsection{Gradient and direction cost}

When the gradient and the down slope direction are used as a cost, the product of both values is applied as a cost. With the function of GIS software, the largest gradient of a cell can be obtained. The number of the cells surrounding a cell is eight. The gradient to each surrounding cell is calculated by the difference of the elevation and the distance between cells. Then the largest gradient is registered in the central cell. Since the absolute difference of elevations is used, only the measure of gradient is obtained and the discrimination between upward and downward gradients cannot be identified by the function. However, the high gradient means that the cell locates on the steep slope. From the point of view of safety and workability, such a place has to be avoided. It is, therefore, expected that the gentle slope is selected by the function of the cost weighted distance if the gradient is used as a cost.

As mentioned above, the slope direction is not known from the above information. The function of the down slope direction in GIS software is, then, used. This function can identify the direction of the largest downward slope. Since the direction is expressed by the angle from north, $\theta$, the angle has to be modified to the angle from the direction to the arrival point from the starting point. Let the angle of the direction from the starting point to the arrival point from north be $\alpha$ as shown in Figure 5. The modified direction is evaluated as $1-\cos (\theta-\alpha)$. By this modification, the value becomes positive and small when the downward slope directed to the arrival point.

The cost is calculated by multiplying the gradient by the above direction. By this treatment, the route having the downward direction to the arrival point and the gentle slope is selected.

\subsubsection{Combination cost}

The combination of both above the costs is also examined. The absolute value of the elevation is quite different from that of the product of the gradient and the direction mentioned above. Therefore, the following normalized cost by the standard deviation is used;

$$
Z=\frac{X-\mu}{\sigma}
$$

where $X$ is each cost, $\mu$ is the average of the cost and $\sigma$ is the standard deviation. The range of both normalized costs becomes similar. As a combination cost, sum of both normalized costs is used to determine the route. However, the value given by (1) has a negative value and the combined cost also becomes negative in some cases. As mentioned earlier, the cost has to be positive to calculate the cost weighted distance. To avoid the negative value of the com-

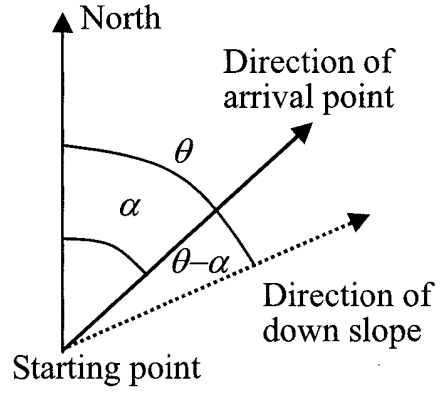

Figure 5: Direction from starting point to arrival one

bination cost, the smallest value of the combination cost is obtained beforehand. Then, the absolute value of the smallest combination cost is added to each combination cost.

\section{Conditions of safe route}

\subsection{General conditions}

To transport water through an irrigation water supply line, some conditions have to be satisfied. According to the criteria of an irrigation pipeline (Japanese Society of Irrigation, Drainage and Rural Engineering, 2000), the followings are written as conditions:

1) A pipeline location is preferable under the hydraulic gradient line;

2) A short pipeline is preferable. However, since the water pressure becomes large at the low location of the undulation, it is preferable to avoid the undulation part. The water pressure over the strength of the pipe has to be avoided;

3) The pipeline gets across a road, a river and a rail track at rights;

4) A soft ground and a confined groundwater have to be avoided;

5) It is preferable that the pipeline is constructed along a road or a boundary of a farm land;

6) It is preferable that the amount of the excavated soil is the mostly same as that of the filling soil; and

7) The irrigation canal is located at the higher place than the drainage one.

Since the temporary irrigation water supply line has to be constructed economically and rapidly, the facility is built on the ground surface and excavation works have to be avoided. Thus, 3), 4) and 6) are not related to the temporary works. Since the water supply line examined in the study is used for only irrigation use, 7) is also not related to this study. The in-service period is expected to be relatively short and a temporary pipeline needs less maintenance than normal pipeline. Thus, 5) does not need to be considered. Among the above conditions, 1) and 2) are the conditions of the temporary water supply line.

If the siphon is designed, the difference between the elevation of the pipe and the piezometric head has to be below $8 \mathrm{~m}$. 


\subsection{Conditions of temporary line}

\subsubsection{Location under hydraulic gradient line}

Since the temporary irrigation water supply line is built on the ground surface, the location of the line is constrained by the topography. Thus, the condition 1) is difficult to be satisfied. Excavation works are needed to conform to the condition.

If the location of the line exists over the hydraulic gradient line, the line becomes a siphon. In this case, the negative pressure head in the tube should not be larger than $-8 \mathrm{~m}$. The piezometric head at an arbitrary location between the starting and arrival point is given by the left hand side of the following equation;

$$
H_{c}+\frac{p_{c}}{\rho g}=H_{a}-\frac{V^{2}}{2 g}-h_{f}
$$

in which $H_{c}$ is the elevation at the arbitrary location, $p_{c}$ is the pressure at the location, $\rho$ is the fluid density, $g$ is the gravitational acceleration, $H_{a}$ is the elevation at the starting point, $V$ is the velocity in the tube and $h_{f}$ is the friction loss head. $h_{f}$ is given by

$$
h_{f}=10.67 C^{-1.85} \cdot D^{-4.87} \cdot Q^{1.85} \cdot L
$$

where $C$ is the velocity coefficient, $D$ is the diameter of tube, $Q$ is the flow rate, $L$ is the distance from the starting point. Since the other loss heads are relatively smaller than the friction loss head, only the friction loss head is considered for simplicity in this study. When the material of the tube is polyethylene, which is tolerant for abrasion and impact, and lightweight, $C$ is 150 . While the values are used as $C$ in this study, the various materials have been used as a temporary water tube. $V$ is obtained by $4 Q / \pi D^{2}$. $Q$ is given by the demand of users and $D$ is calculated by

$$
H_{a}-H_{b}=\frac{\left(4 Q / \pi D^{2}\right)^{2}}{2 g}+10.67 C^{-1.85} \cdot D^{-4.87} \cdot Q^{1.85} \cdot L^{\prime}(4)
$$

where $H_{b}$ is the elevation of the arrival point. In this equation, $L$ ' is the distance between the starting and arrival point. $V\left(=4 Q / \pi D^{2}\right)$ is calculated by using $D$ given by (4).

By using $V$ determined from the above process, the piezometric head at the arbitrary location is obtained from (2). This piezometric head constitutes the hydraulic gradient line. If the velocity is changed, the hydraulic gradient line is also changed. If the elevation at the location is higher than the piezometric head, the pressure in the tube at the location becomes negative, meaning the siphon. If the elevation is higher than the piezometric head by more than $8 \mathrm{~m}$, the cost of the cell, which was explained in 3.1 , is set at the high value to avoid that location in the subsequent iterative examination. This is because water cannot be supplied if the siphon does not work well.

When the elevation is lower than the piezometric head, the pressure in the tube becomes positive and the line exists below the hydraulic gradient line. Since the general limita- tion of the positive pressure is $1 \mathrm{MPa}$, the same treatment to avoid the critical location as the siphon has to be carried out, if the positive pressure is larger than $1 \mathrm{MPa}$. If the positive pressure is not so high, it is preferable that the length of the part under the hydraulic gradient line is large. Therefore, the following index is used to examine the safety;

$$
\text { index_1 }=\frac{\text { Number of cells under pizometric head }}{\text { Total number of cells in route }}
$$

If this index is close to 1 , the safety of the facility becomes large.

\subsubsection{Length of line}

From the condition 2), it is preferable that the route is a linear line. It is, however, impossible for a temporary irrigation water supply line. As an index of the linearity, the following index is examined;

$$
\text { index_2 }=\frac{\text { Direct distance }}{\text { Actual distance }}
$$

If index_ 2 is close to 1 , the line is evaluated to be economical.

\subsubsection{Undulation of line}

As mentioned earlier, the temporary irrigation water supply line will be constructed on the ground surface. Therefore, the undulation of the ground has a large effect on the route configuration. From the point of view of the workability to construct the facility, it is preferable that the undulation is small. The small undulation is also desired for the safety of the tube. In this study, the idealized route is assumed as a quadratic curve from starting point, which is obtained by fitting to the vertical section of determined route. The deviation of the elevation of the route from the quadratic

Table 1: Examination cases and its elevation and length (m)

\begin{tabular}{cccc}
\hline Case & Starting point & Arrival point & Direct distance \\
\hline $\mathrm{AB}$ & 244 & 75 & 2086 \\
$\mathrm{CD}$ & 85 & 28 & 1833 \\
$\mathrm{EF}$ & 179 & 163 & 1386 \\
$\mathrm{GH}$ & 19 & 5 & 760 \\
\hline
\end{tabular}

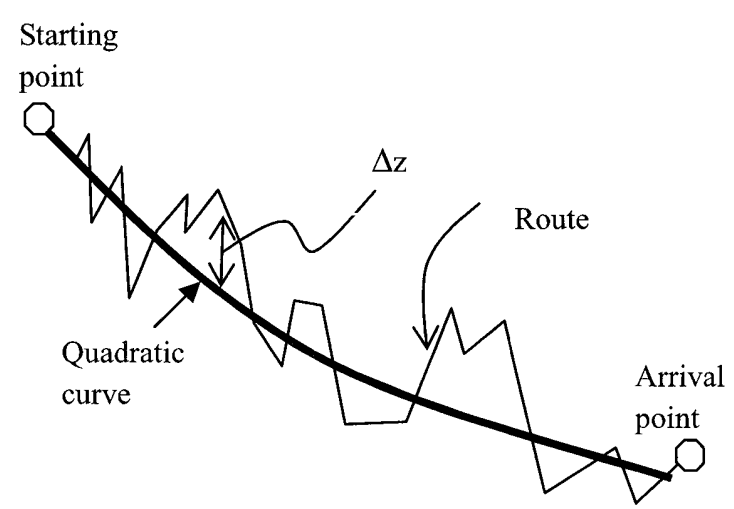

Figure 6: Deviation from gradient line 
curve from the starting point is evaluated to select a preferable route. Figure 6 shows the schematic view of the vertical section of the line and the deviation of the elevation from the quadratic curve by $\Delta z$. The root mean the square of $\Delta z$ is the third index, index_3. If this value is small, the workability to construct the route is high.

\section{Examination results}

\subsection{Examination cases}

Table 1 shows the examined cases and the elevation of each starting and arrival point. The direct distance is also indicated. The locations of A-H are shown in Figure 1. Cases $\mathrm{AB}$ and $\mathrm{EF}$ are the cases at the high elevation. The difference of the elevation between starting and arrival points is large in Case $\mathrm{AB}$, while that of Case EF is small. Cases $\mathrm{CD}$ and $\mathrm{GH}$ are the cases at the low elevation. The difference of the elevation between each starting and arrival point of Case $\mathrm{CD}$ is relatively larger than that of Case $\mathrm{GH}$.

As mentioned earlier, these starting and arrival points do not coincide with the actual situation. These points are selected to examine the proposed method. To examine the effect of velocity, two demands of water supply are assumed; $0.5 \mathrm{~m}^{3} / \mathrm{s}$ and $0.05 \mathrm{~m}^{3} / \mathrm{s}$.

\subsection{Examination results}

Figures 7-10 show the determined route from the methods using each cost mentioned in 3.2. If the average velocity is high, the hydraulic gradient line lowers. If the change in the hydraulic gradient line affects the siphon design, the route is changed with dependency of the velocity. In the examination cases, only Case $\mathrm{AB}$ needed the iterative selection. However, the difference by the demands of water supply did not have an influence on the route selection. Therefore, the routes obtained from the examination were unique for each case.

It can be seen for the horizontal configurations that the method using the elevation cost gives the rectilinear route for all cases. On the other hand, the method using the gradient and direction cost gives the zigzag route. In Cases $A B$ and $\mathrm{EF}$ which are the cases at the high elevation, every method avoids the ridge and selects the valley. In Cases CD and $\mathrm{GH}$ which are the ones at the low elevation, all routes are mostly rectilinear.

It is found from the vertical configuration that the gradient and direction cost gives the route having the high elevation and the long distance. Therefore, the average gradient of the route evaluated from the gradient and direction cost is gentle. In Case EF, since the route went through the valley, the route had a back siphon before reaching at the arrival point in all methods.

Table 2 shows the values of the indexes introduced in the previous chapter. It is observed that the elevation cost gives the high values for index_1 and index_2. In particular, the values of index_2 from the elevation cost are the highest of all cases. The difference of index_1 among the costs is relatively small in comparison with that of index_2. When the demand of water supply is small, the value of index 1 becomes large in Cases $\mathrm{CD}$ and $\mathrm{GH}$, and the safety is in- creased. Since index_1 shows the values over 0.9 for most results, it is found that the selected routes exist mostly under the hydraulic gradient line. On the other hand, index 2 shows the relatively large difference among the costs. The length of the route from the gradient and direction cost becomes longer by about $25 \%$ than that of the elevation cost. Index_2 by the combination cost is intermediate of the elevation cost and the gradient and direction cost. Index 3 is diverse among the cases. This is because the approximation accuracy of the quadratic curve fitted to the selected route is diverse. However, as a tendency, the elevation cost gives the large undulation in the case where the difference of the elevation between starting and arrival points is large as Cases $\mathrm{AB}$ and $\mathrm{CD}$. In the case where the difference of the

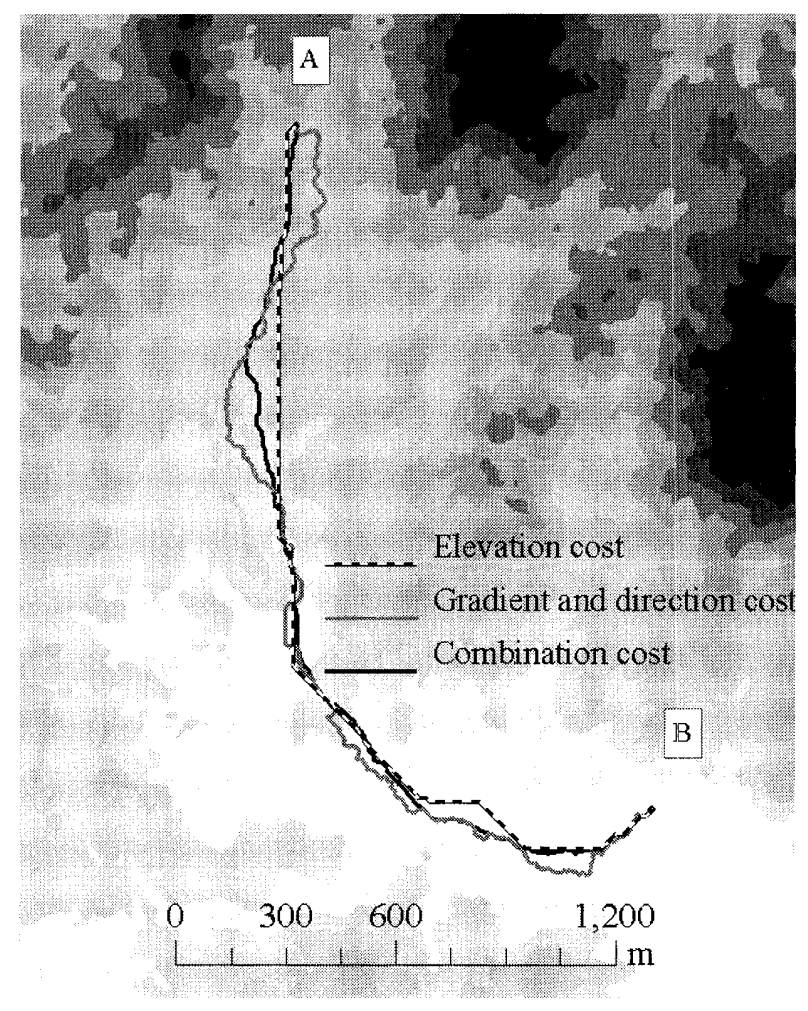

(a) Horizontal section

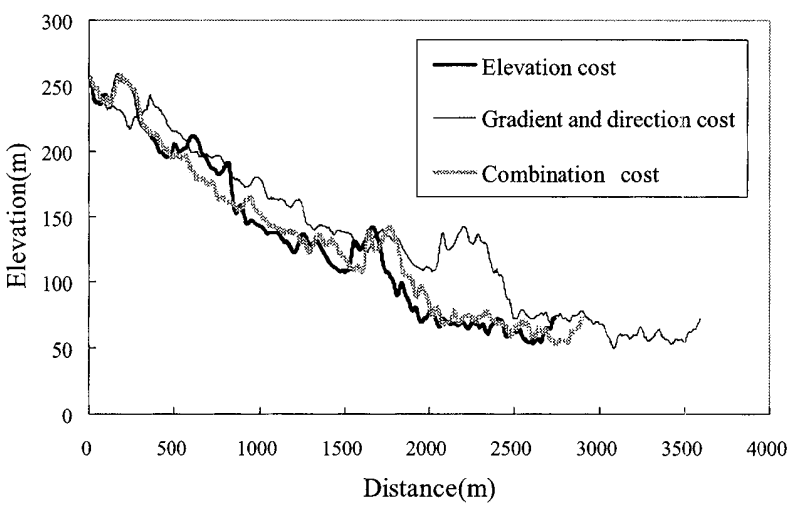

(b) Vertical section

Figure 7: Routes of Case AB 
elevation is small like Cases EF and GH, the gradient and direction cost gives the large undulation.

It can be concluded that the elevation cost gives the short

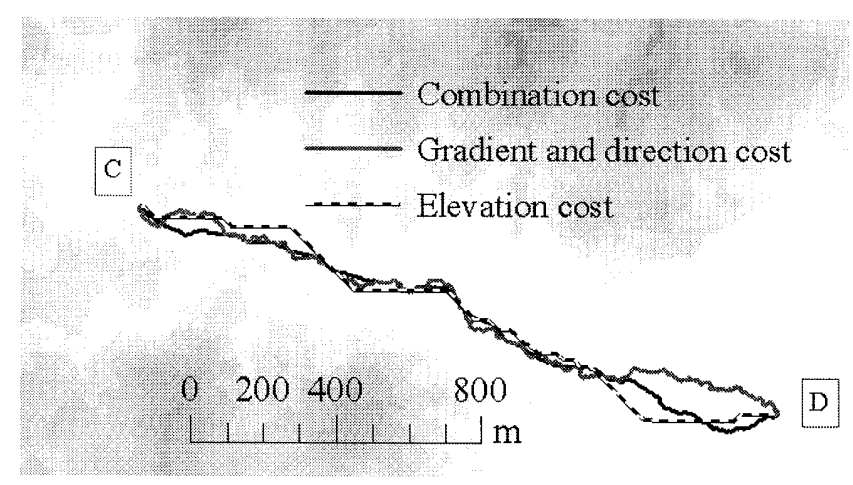

(a) Horizontal section

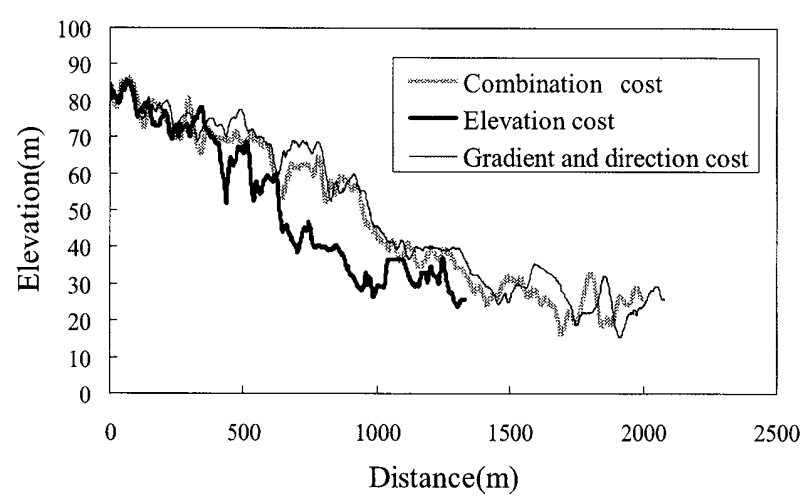

(b) Vertical section

Figure 8: Routes of Case CD

\section{G}

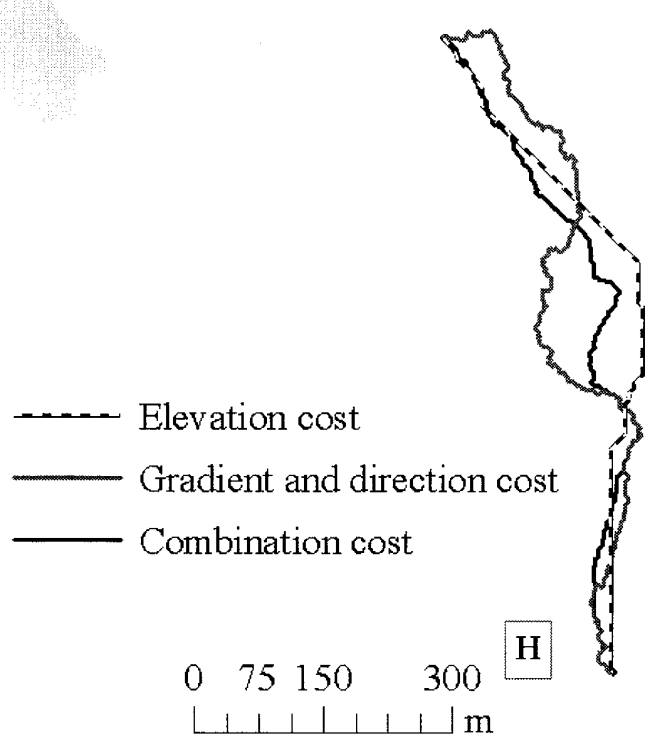

(a) Horizontal section distance and the gradient and direction cost gives the gentle descent of the route. The combination cost gives the intermediate results of both costs.

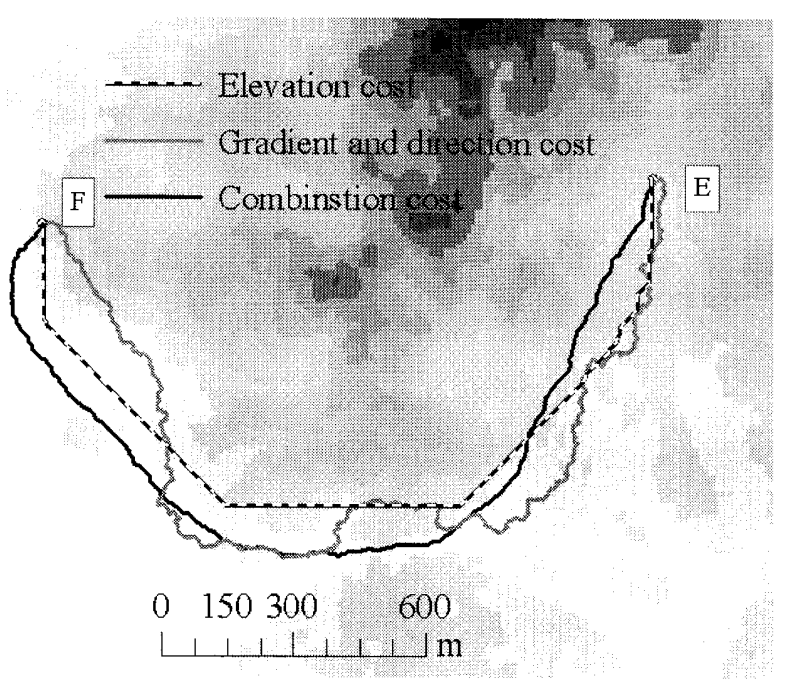

(a) Horizontal section

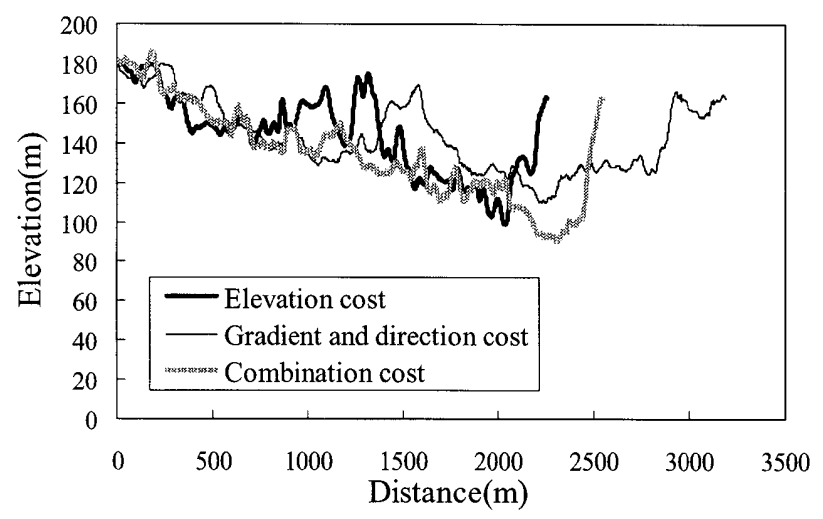

(b) Vertical section

Figure 9: Routes of Case EF

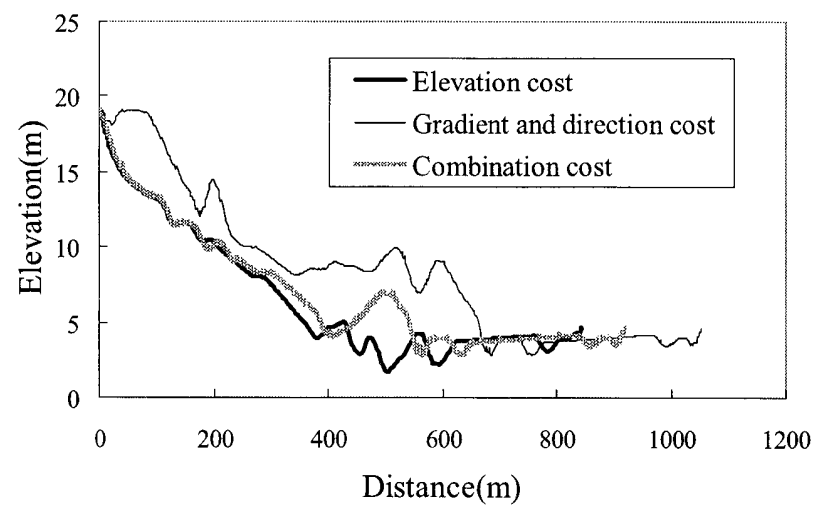

(b) Vertical section

Figure 10: Routes of Case GH 
Table 2: Value of each index

\begin{tabular}{|c|c|c|c|c|c|c|c|}
\hline \multirow{2}{*}{ Case } & \multirow{2}{*}{ Cost } & \multicolumn{2}{|c|}{ Index_1 } & \multicolumn{2}{|c|}{ Index_2 } & \multicolumn{2}{|c|}{ Index_3 } \\
\hline & & $0.5^{*}$ & $0.05 *$ & $0.5^{*}$ & $0.05^{*}$ & $0.5^{*}$ & $0.05^{*}$ \\
\hline \multirow{3}{*}{$\mathrm{AB}$} & Elevation & 0.96 & 0.96 & 0.76 & 0.76 & 18.0 & 18.0 \\
\hline & Gradient and direction & 0.99 & 0.99 & 0.58 & 0.58 & 11.5 & 11.5 \\
\hline & Combination & 0.90 & 0.90 & 0.72 & 0.72 & 16.1 & 16.1 \\
\hline \multirow{3}{*}{$\mathrm{CD}$} & Elevation & 0.92 & 0.94 & 0.92 & 0.92 & 16.2 & 16.2 \\
\hline & Gradient and direction & 0.76 & 0.80 & 0.77 & 0.77 & 9.7 & 9.7 \\
\hline & Combination & 0.80 & 0.86 & 0.88 & 0.88 & 5.9 & 5.9 \\
\hline \multirow{3}{*}{$\mathrm{EF}$} & Elevation & 1.00 & 1.00 & 0.61 & 0.61 & 13.1 & 13.1 \\
\hline & Gradient and direction & 0.95 & 0.95 & 0.43 & 0.43 & 15.8 & 15.8 \\
\hline & Combination & 0.95 & 0.95 & 0.54 & 0.54 & 10.3 & 10.3 \\
\hline \multirow{3}{*}{$\mathrm{GH}$} & Elevation & 0.99 & 1.00 & 0.90 & 0.90 & 1.7 & 1.7 \\
\hline & Gradient and direction & 0.91 & 0.92 & 0.72 & 0.72 & 2.3 & 2.3 \\
\hline & Combination & 0.99 & 1.00 & 0.83 & 0.83 & 2.1 & 2.1 \\
\hline
\end{tabular}

\section{Conclusions}

In this paper, the method to determine the route of a temporary irrigation water supply line with GIS was introduced. In the emergency situation, the temporary facility for water supply has to be constructed rapidly and economically. For this objective, by assuming that the facility will be constructed on the ground surface without excavation works and the gravity irrigation will be adopted to transport water, the route selection method was examined with GIS. Moreover, to determine the route easily and quickly, the function in the software was used as much as possible. The cost weighted distance of the function was mainly used, whose function considered the cost and selected the most economical route. In this study, the elevation, the gradient and direction and the combination of both values were applied as a cost to determine the safe and economical route of a temporary irrigation water supply line. To examine the selected route, three indexes were introduced. As a result, the followings are concluded:

1) When the elevation cost was used, the distance became the smallest. This indicated that the economical route was selected. However, the undulation of the route in the case where the difference of the elevation between starting and arrival points was large was generally large and the mean gradient was also large. Therefore, the construction of the route would be difficult.

2) When the gradient and direction cost was used, the distance became the longest and the mean gradient was small. While the route was not economically effective, the construction of the line would be easy.

3) The combination cost gave the mostly intermediate results of both costs.

When the water supply line is constructed, the actual situation of the ground surface and vegetation has to be considered. In the process to select the route with GIS software, only the procedure to modify the direction has to be carried out by the user. The other procedures can be carried out by using the function of the software. Therefore, if the DEM of a region is prepared, the above examination can be done relatively easily, while the hydraulic examination has to be carried out. Most economical and safe routes can be chosen from the examined results and the actual situation.

\section{References}

[1] Inoue K., Nakanishi N., Nakazato H. and Naka T. (2007): Construction of history management system using GIS for large-scale agricultural pipe line - for priority decision support system of function maintenance-, Technical Report of The National Institute for Rural Engineering, 296, pp.51-63. (in Japanese).

[2] Japanese Society of Irrigation, Drainage and Rural Engineering (2000), Handbook of Agricultural Engineering, pp.455-456. (in Japanese).

[3] Japanese Society of Irrigation, Drainage and Reclamation Engineering, Kyoto branch (2005): Report of the disaster at Awaji Island by typhoon No. 16 in 2004. (in Japanese).

[4] Naka T., Gotou M., Taruya H., Tanaka Y., Yoshinaga I. and Hata K. (2006): Field observation on hydraulic damages by the mid Niigata prefecture earthquake in 2004 and disaster rehabilitations in Shinanogawa-sagan irrigation project, Technical Report of The National Institute for Rural Engineering, 202, pp.25-36. (in Japanese).

[5] Niigata prefecture homepage: http://www.pref.niigata.jp /seisaku/kokusai/english/.

[6] Scott, C., Bob, B.and Andy, M. (2003): Arc GIS 8. Tokyo. ESRI Japan, Ltd..

[7] Tanaka Y., Shimazaki M., Shima T. and Naka T. (2002): Object oriented development of a planning aid system for line selections of irrigation pipeline systems, Technical Report of The National Institute for Rural Engineering, 200, pp.63-74. (in Japanese). 